\title{
Ultra-Precision Machining of a Large Amplitude Sinusoidal Ring Surface Based on a Slow Tool Servo
}

\author{
Shijun Ji - Huijuan Yu - Ji Zhao* - Xiaolong Liu - Mingxu Zhao \\ Jilin University, School of Mechanical Science and Engineering, China
}

A sinusoidal ring surface with a large size, amplitudes in the macro-level, sub-micrometre form accuracy and nanometre surface roughness plays an important role in modern optical systems and precision calibration, but it is difficult to fabricate with traditional cutting methods. A sinusoidal ring surface with a submillimetre amplitude, machined using a slow tool servo (STS) assisted by single-point diamond turning (SPDT) is presented in this paper. The kinematic characteristic of machine axes is analysed to assess the feasibility of the turning process. The tool path generation, tool geometry optimization, and tool radius compensation are investigated for fabrication of the desired surface. $A$ fabricating experiment of a $0.4 \mathrm{~mm}$ amplitude sinusoidal ring surface is performed, and the surface profile is measured with a Talysurf PGI 1240, a measurement system for the small to medium sized aspheric optics. After dealing with original measured curves, a form accuracy $0.274 \mu \mathrm{m}$ in peak-to-valley error (PV) and surface roughness $7.5 \mathrm{~nm}$ in Ra are obtained for the machined surface, and it can be seen that STS can be used for machining of a large amplitude sinusoidal ring surface.

Keywords: sinusoidal ring surface, STS, kinematic characteristic, ultra-precision machining

Highlights

- A sinusoidal ring surface with a submillimetre amplitude is machined using a slow tool servo (STS) assisted by single-point diamond turning (SPDT).

- The kinematic characteristic of machine axes is analysed to assess the feasibility of the turning process.

- Analysis about tool path generation and tool radius compensation, and optimization of the cutting tool parameters are necessary before the actual cutting experiment.

- A form accuracy $0.274 \mu \mathrm{m}$ in peak-to-valley error (PV) and surface roughness $7.5 \mathrm{~nm}$ in Ra are obtained for the machined surface.

\section{O INTRODUCTION}

Freeform surfaces can make a smaller, lighter, higherperformance optical system and have been the subject of increased attention in recent years [1] to [3]. The sinusoidal ring surface is a typical shape of freeform surfaces, which can be used in positioning multi-axis precision motion stages and as aerospace components. The surfaces are required to be of large size, and have sub-micrometre form accuracy and a nanometre surface finish in those applications [4] and [5].

Although the sinusoidal ring surface is a rotationally symmetric surface, the continuously changing position and moving in the $\mathrm{Z}$ direction still make the processing difficult to perform while the workpiece is turned with a spindle. Several methods can be used to manufacture the sinusoidal ring surface, such as grinding, polishing and lapping. However, these methods use multiple processes, and it is not easy for them to fabricate complex surfaces, such as sinusoidal surfaces with high form accuracy and highly smooth surfaces [6]. Presently, SPDT with fast tool servo (FTS) and STS are becoming pre-eminent due to their ability to achieve optical quality in a one-stage process without subsequent processing [7] and [8]. As for FTS, the cutting tool can be rapidly and accurately positioned in the manufacturing process; therefore, a freeform surface with micro/nano-structures can be fabricated. However, the amplitude of the swing is usually small, which cannot be used in large sagittal surface machining. For example, $\mathrm{Lu}$ et al. [9] made a sinusoidal grid surface using a fast tool servo system with a profile wavelength is $350 \mu \mathrm{m}$, and a peak-tovalley amplitude of approximately $16 \mu \mathrm{m}$. Rasmussen et al. [10] designed a piezoelectric-driven cutting tool system, which can dynamically control the depth of cut; it can be used to machine slightly non-circular workpieces. With an amplification mechanism, the tool was able to produce a $52 \mu \mathrm{m}$ travel range with a frequency of $200 \mathrm{~Hz}$. As for STS, all axes can be under full coordinate position control. Moreover, the form accuracy and the quality of a machined surface depend on the proper selection of the tool path, cutting strategies and cutting parameters [11] to [13]. Several papers have examined sinusoidal surfaces in recent years. For instance, Zhang et al. [1] presented studies on the sinusoidal surface machining with a proposed cylindrical coordinate method using the STS. With the proposed method, a $40 \mu \mathrm{m}$ amplitude sinusoidal surface with a nanometric finish of $5.54 \mathrm{~nm}$ in $\mathrm{Ra}$ is achieved. Gao et al. [6] manufactured a sinusoidal angle grid surface with a wavelength of $100 \mu \mathrm{m}$ and 
an amplitude of $100 \mathrm{~nm}$ over a large surface area with a diameter of $150 \mathrm{~mm}$. Zhu et al. [14] described the manufacturing process of a sinusoidal grid surface using FTS on a Zr-based bulk metallic glass (BMG); an $80 \mu \mathrm{m}$ amplitude sinusoidal surface is obtained using diamond cutting tools.

The challenge of manufacturing a submillimetre amplitude sinusoidal ring surface using a three-axis $(X, Z, C)$ turning machine is to achieve the ultraprecision form accuracy [15] and [16]. Thus, this paper presents a theoretical research on the machining of sinusoidal ring surface using the STS, including tool path generation, kinematic characteristic of two linear axes and the selection of cutting tool geometry. Then, a typical sinusoidal ring surface is machined with STS to demonstrate the feasibility of the theoretical analyses.

\section{TOOL PATH GENERATION}

In single-point diamond turning, a workpiece is mounted on the spindle, and the schematic diagram of cutting the sinusoidal ring surface using STS is shown in Fig. 1, where a and $h$ are the wavelength and the double amplitude of the sinusoidal surface, respectively. The sinusoidal ring surface in cylindrical coordinate system $(X, \theta, Z)$ can be expressed as:

$$
\left\{\begin{array}{c}
z=\frac{h}{2} \sin \left(\frac{2 \pi}{a} x\right)+z_{0} \\
x^{2} \leq\left(\frac{D}{2}\right)^{2}
\end{array},\right.
$$

where $D$ is the maximum diameter of the sinusoidal ring surface.

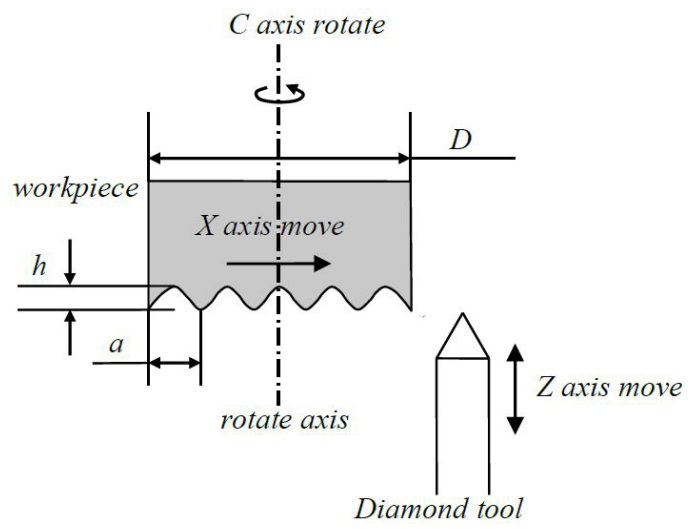

Fig. 1. Schematic diagram of cutting a sinusoidal ring surface

The diamond tool is moved along the $Z$ direction in coordination with the movement of the workpiece along the $X$ direction in the single-point diamond turning process. To obtain the continuous and smooth surface, the tool path of the diamond tool must be accurately generated in advance, and a planar spiral is used to generate the cutting tool path. The path of tool contact points is obtained subsequently by projecting the planar spiral on the desired surface in the $Z$ direction. The planar spiral curve is related to the cutting parameters [14], such as feed rate $f[\mathrm{~mm} /$ $\mathrm{min}]$ and spindle speed $v[\mathrm{rev} / \mathrm{min}]$. The expression in polar coordinates can be shown as follows:

$$
x=\frac{f}{2 \pi \nu} \theta, \quad\left(x \leq \frac{D}{2}\right) .
$$

According to Eqs. (1) and (2), the calculated path of tool contact points for the sinusoidal ring surface can be illustrated by Fig. 2 .

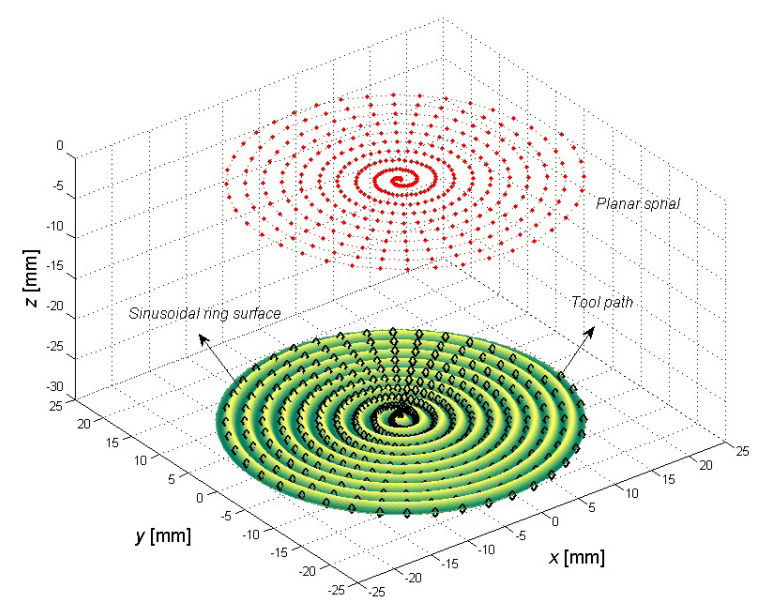

Fig. 2. Path of tool contact points for sinusoidal ring surface

To avoid the influence of tool radius and to obtain a surface with superior form accuracy, the geometry of the diamond tool nose must be considered, since it is not an ideal point but a half-cylinder with radius $R$ [17] and [18]. In fact, by projecting points of the planar spiral on the desired surface, cutting contact points can then be obtained. In order to generate the motion commanders of $\mathrm{CNC}$ machine tools, the cutting contact points must be converted into cutter location points; that is to say, the tool radius compensation must be conducted. Since every cutting contact point has a unique curvature along the cutting trajectory on the machining surface, the tool compensation can be achieved by offsetting the tool contact points with a value of tool radius $R$ in the normal vector direction rather than only by offsetting the tool contact points with a value of tool radius $R$ in the $Z$ direction. The 
a)
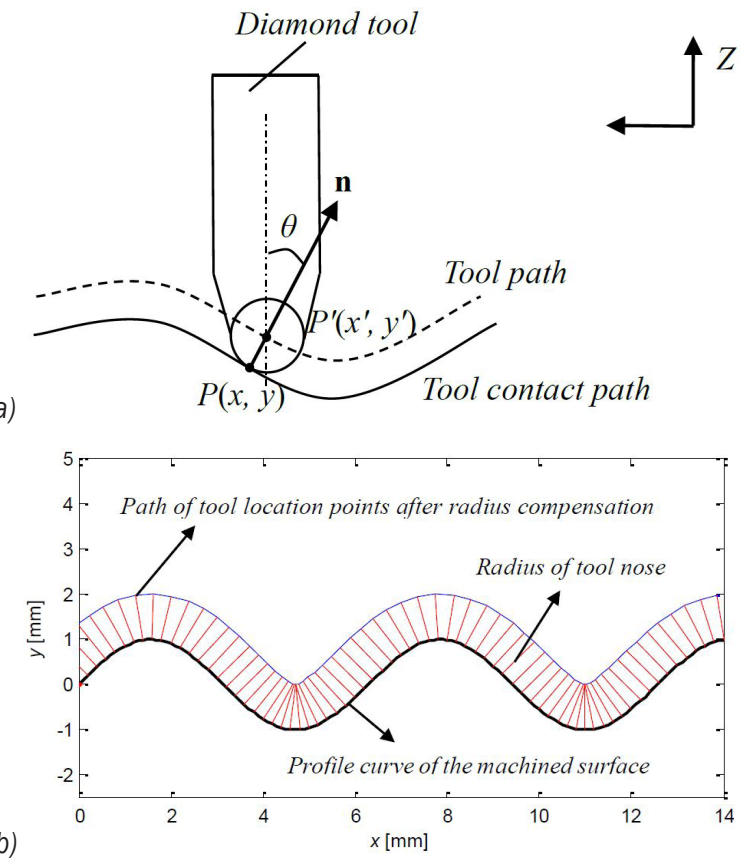

Fig. 3. Tool radius compensation a) the schematic diagram of tool radius compensation b) tool radius compensation for sinusoidal ring surface

schematic diagram of tool radius compensation is illustrated in Fig. 3, where point $P(x, y)$ is an arbitrary point on the tool cutting contact path. The normal vector $\mathbf{n}$ at point $P$ on the tool cutting contact path determined the compensation direction, and the offset value is the tool nose radius $R$; then, $P^{\prime}\left(x^{\prime}, y^{\prime}\right)$ is the corresponding compensation tool location point. All of the compensation tool locational points constitute the tool location path. The tool location point can be calculated by:

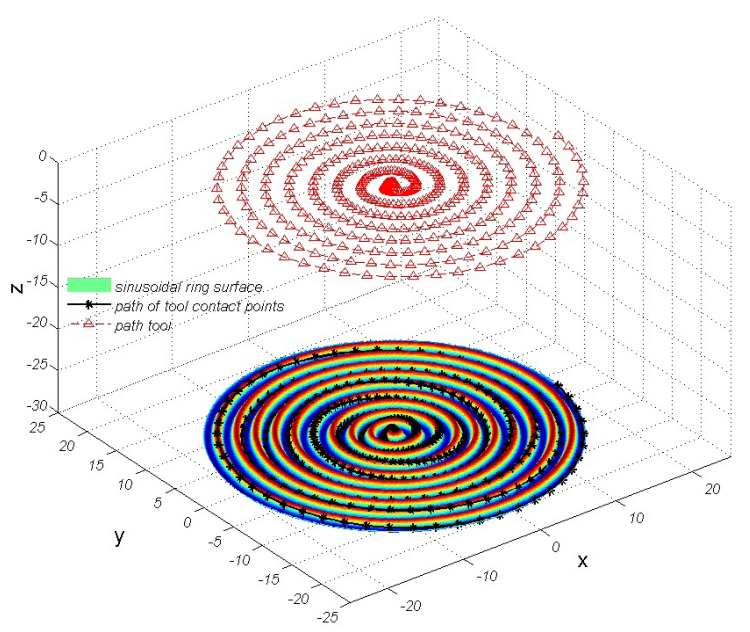

Fig. 4. Tool path for sinusoidal ring surface in $3 D$

$$
\left\{\begin{array}{l}
x^{\prime}=x+R \sin \theta \\
y^{\prime}=y+R \cos \theta
\end{array},\right.
$$

where $\theta$ is the angle between normal vector $\mathbf{n}$ and perpendicular line. After tool radius compensation with Eq. (3), Fig. 4 shows the final tool location path of sinusoidal ring surface, which drives the diamond tool accurately in a ultra-precision machine.

\section{ANALYSIS OF KINEMATIC CHARACTERISTICS}

Since the machining efficiency is greatly influenced by the velocity and accelerated speed limitation of every axis, it is necessary to analyse the kinematic characteristic of every axis before cutting. The movement of cutting point in $X$ axis and $Z$ axis along with the turning of $C$ axis is illustrated in Fig. 5 under the $X-\theta-Z$ coordinate system.

To analyse the kinematic characteristic of the fabricating process, the cutting surface has been stretched out as shown by Fig. 5. Obviously, the expanded shape of the sinusoidal ring surface is a straight sinusoidal wave surface. The point $P_{i}$ is an arbitrary cutting point on the cutter path, and the displacement of $X$ axis to the cutting point is a linear relation with the angular displacement $\theta$ in $X-\theta-Z$ coordinate system. While, the relation between displacement of $Z$ axis and angular $\theta$ can be indicated by:

$$
z=z(\theta)=\frac{h}{2} \sin \left(\frac{f}{a v} \theta\right)+z_{0} .
$$

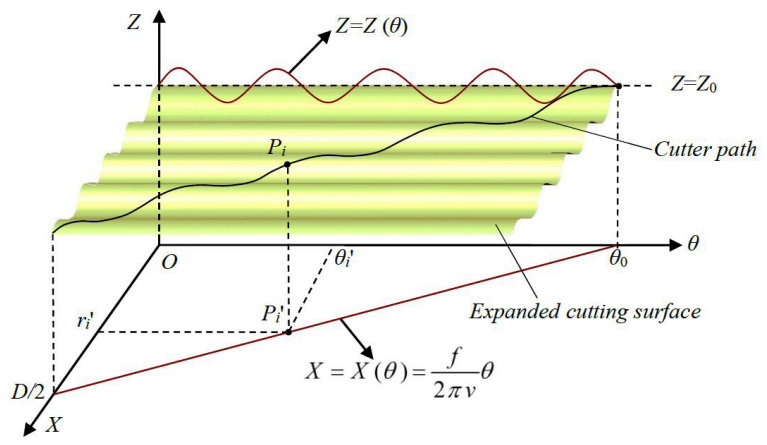

Fig. 5. The kinematic analysis of diamond tool

As indicated by Eq. (4), the displacement of $Z$ axis of the cutting point is a sinusoidal function of angular displacement $\theta$, and the amplitude of the sinusoidal function is constant $h / 2$; therefore, the wavelength is changed to $2 \pi a v / f$. Moreover, it also can be seen that the position and moving direction in the $Z$ direction are continuously changeable with the 
change of angular displacement $\theta$, so the STS of $Z$ axis is inevitably used to provide accurate displacement control. The speed and acceleration of the $X$ and $Z$ axes can be obtained by calculating the first derivative and second derivative of displacement function $X=X(\theta)$ and $Z=Z(\theta)$, respectively. The maximum velocity and maximum acceleration of linear axes should not exceed the corresponding maximum value of the machine tool, which are $4000 \mathrm{~mm} / \mathrm{min}$ and $1980 \mathrm{~mm} /$ $\mathrm{s}^{2}$ for the Nanoform 250 machine tool.

\section{PARAMETER SELECTION OF DIAMOND TOOL}

In the ultra-precision turning process, the proper selection of the cutting tool plays an important role in the processing planning, since the quality of the final product is greatly influenced by the diamond tool geometry [19] to [21]. Thus, cutting tool geometry must be optimized according to practical cutting conditions. [22].

The cutting tool radius selection of the diamond tool is presented in Fig. 6, which is based on the stretch-out view of cutting surface. As shown in Fig. 6a, point $P_{i}$ is an arbitrary cutting point on the tool path, and vector $\mathbf{n}_{t i}$ is the tangent vector for path curve at point $P_{i}$. Point $P_{i}^{\prime}$ and $\mathbf{n}_{t i}^{\prime}$ are the projection point and vector of $P_{i}$ and $\mathbf{n}_{t i}$ on the $X-O-\theta$ plan, respectively. Plane $\gamma$ is given by passing $P^{\prime}{ }_{i}$ and vertical to $\mathbf{n}_{t i}^{\prime}$, and line $l_{r}$ is the intersection line of plane $\gamma$ and the cutting surface. The enlarged partial view of the cutting condition is presented in Fig. 6b, which illustrates that the cutting interference can occur when curvature radius and arc angle of cutting point are both smaller than the corresponding value $R$ and $\theta$ of the diamond tool, such as cutting point $P_{i}$, and the opposite condition is applicable, such as point $P_{k}$. Therefore, the optimal cutting tool radius and included angle should be less than the minimum value of curvature radius and arc angle at all cutting points.

Clearance angle is another indispensable parameter for the selection of tool geometry. As shown in Fig. 7a, plane $\delta$ is established by passing the point $P_{i}^{\prime}$ and vector $\mathbf{n}_{t i}^{\prime}$, and line lc is the intersection line of plane $\delta$ and the cutting surface. The selection of clearance angle at one cutting point is determined by the inclination angle of the tangential line at this point on the cutting curve, and the cutting interference occurs in the case that the clearance angle less than the inclination angle of the tangential line, such as point $Q_{i}$, while the opposite condition point $Q_{j}$ is feasible, as demonstrated in Fig. 7b. With the purpose of avoiding interference at any cutting points, the optimal clearance angle should be selected for machining, and
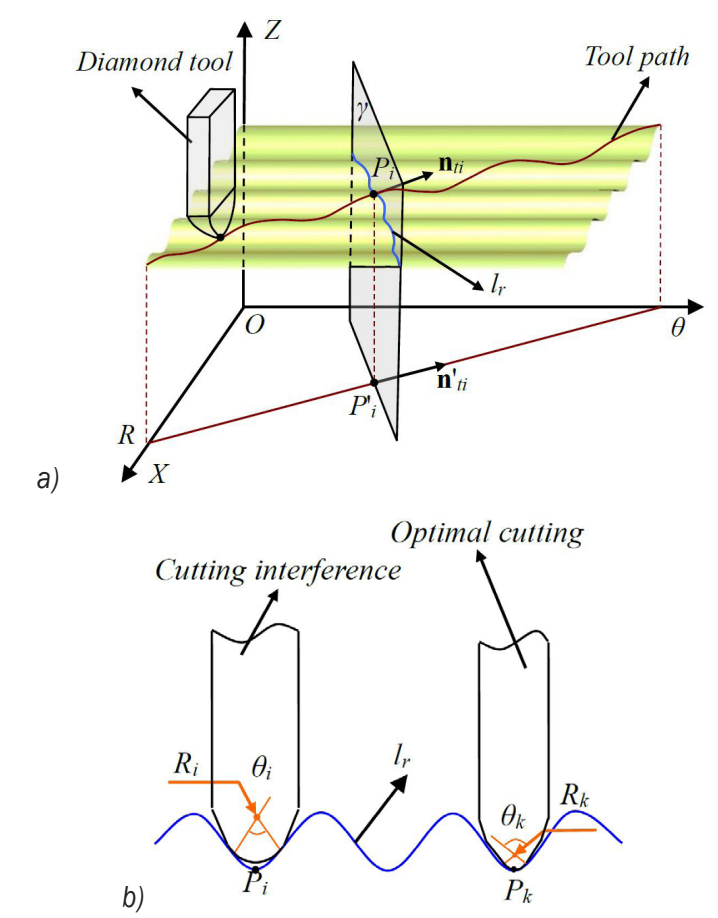

Fig. 6. Optimal tool radius selection; a) schematic diagram of radius selection, and b) partial enlarged detail view
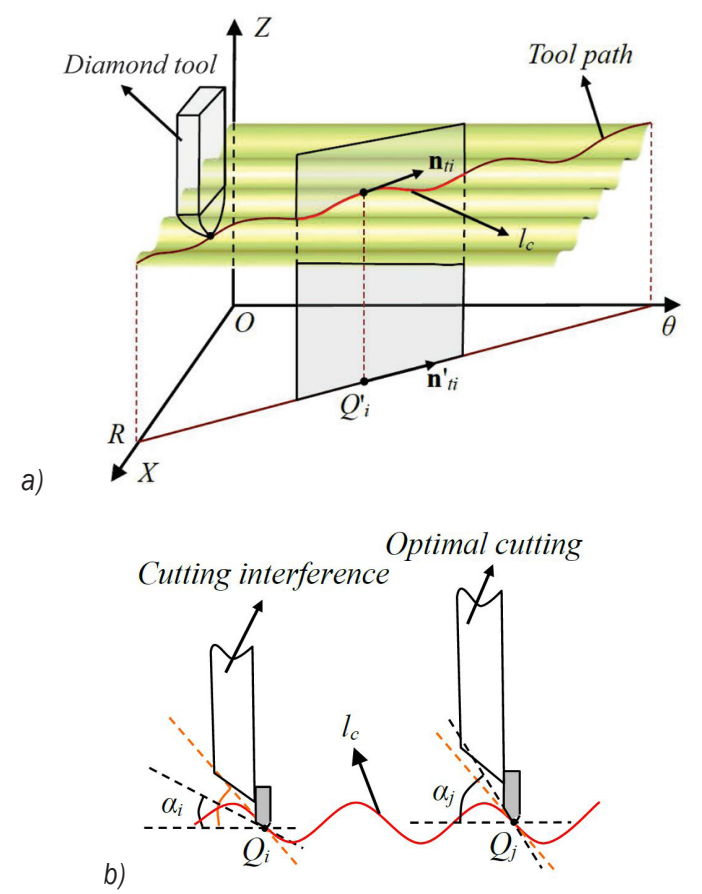

Fig. 7. Optimal clearance angle selection; a) schematic diagram of clearance angle selection, and b) partial enlarged detail view

the complementary angle of the optimal clearance angle must be larger than the maximum value of the inclination angle in all cutting point positions. 


\section{EXPERIMENTS AND DISCUSSION}

A sinusoidal ring surface expressed by Eq. (1) with $h=0.4 \mathrm{~mm}, a=2 \mathrm{~mm}$ and $D=20 \mathrm{~mm}$ was simulated and fabricated in the Nanoform 250 ultra-precision machining system. Both the $X$ and $Z$ axes of the Nanoform 250 are constructed with non-contact oil hydrostatic bearing sideways, non-contact linear motors, and non-contact encoder feedback, liquid cooled systems for slides. In this manner, there is zero stick-slip or friction and thermal stability. The material of the workpiece is Al-6061. The diamond tool with a nose radius of $0.506 \mathrm{~mm}$, an included angle of $120^{\circ}$, and a clearance angle of $10^{\circ}$ was applied, which satisfies the analyses in Section 4. The machining quality on computerized numerically controlled (CNC) machine tools is sensitive to the machining parameters [23]. In this paper, the cutting conditions we adopted are listed in Table 1.

Table 1. Cutting conditions

\begin{tabular}{lc}
\hline Parameters & Values \\
\hline$X$ increment per rev & $0.01 \mathrm{~mm}$ \\
\hline$C$ increment & $1^{\circ}$ \\
\hline Max speed of $X$ axis & $0.011 \mathrm{~mm} / \mathrm{s}$ \\
\hline Max speed of $Z$ axis & $0.0063 \mathrm{~mm} / \mathrm{s}$ \\
\hline Frequency of $Z$ axis & $0.0055 \mathrm{~Hz}$ \\
\hline Acceleration of $X$ and $Z$ axes & $0.2 \mathrm{~g}$ \\
\hline Amplitude of the surface & $0.4 \mathrm{~mm}$ \\
\hline
\end{tabular}

Before ultra-precision manufacturing the workpiece, centring the tool with an LVDT video tool set station and dynamic balancing of the spindle were conducted to ensure high machining precision. The aluminium workpiece was fabricated with STS at a spindle speed of $60 \mathrm{rpm}$. The actual fabrication structure is displayed in Fig. 8. A rotationally symmetrical fixture was applied to connect the vacuum chuck and workpiece. The designed cutting path, as shown in Fig. 4, was employed to perform the cutting experiment.

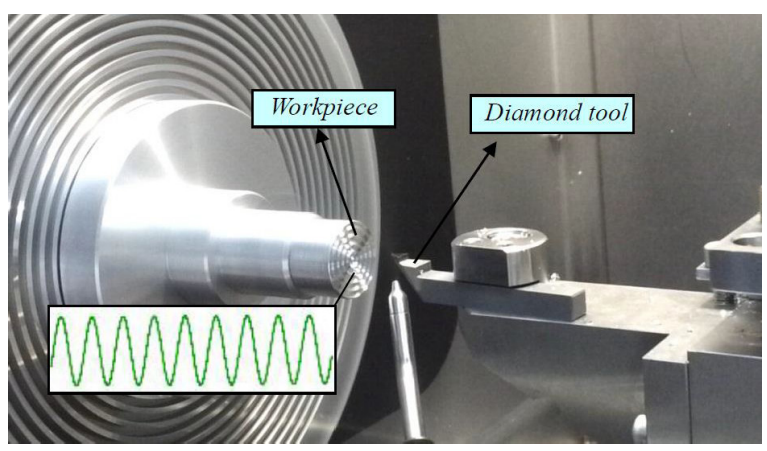

Fig. 8. Photos of in-situ machining

To reveal the surface quality of the machined part, the surface roughness and form accuracy of the machined sinusoidal ring surface were measured and evaluated with a high magnification microscope (Contour GT) and a precision form measurement system (Taylor Hobson), respectively. Firstly, Contour GT, a white-light interferometric microscope, is used to measure surface roughness. The measuring area is a rectangular shape of $60 \mu \mathrm{m} \times 45 \mu \mathrm{m}$, which is about $3 \mathrm{~mm}$ to the outside boundary of the workpiece. In the process of measurement, the

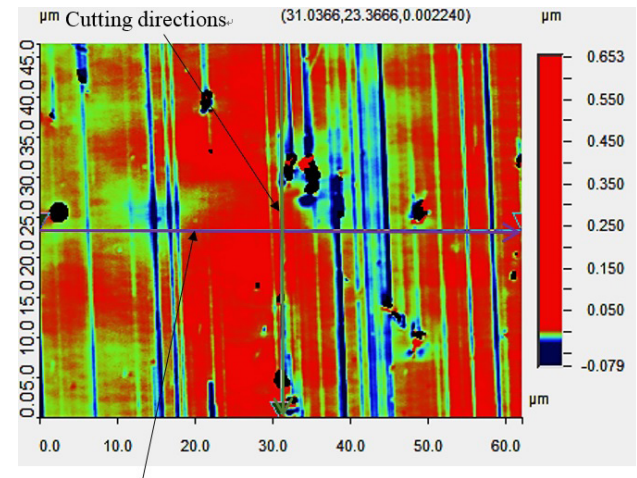

a) Measure directions

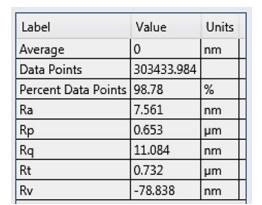

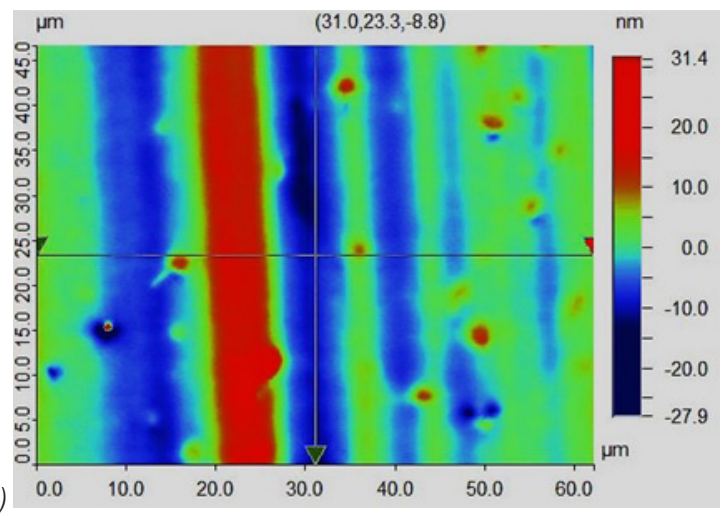

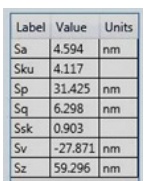

Fig. 9. Measurement result of surface roughness for machined sinusoidal surface: a) linear roughness measur. b) areal roughness measur. 


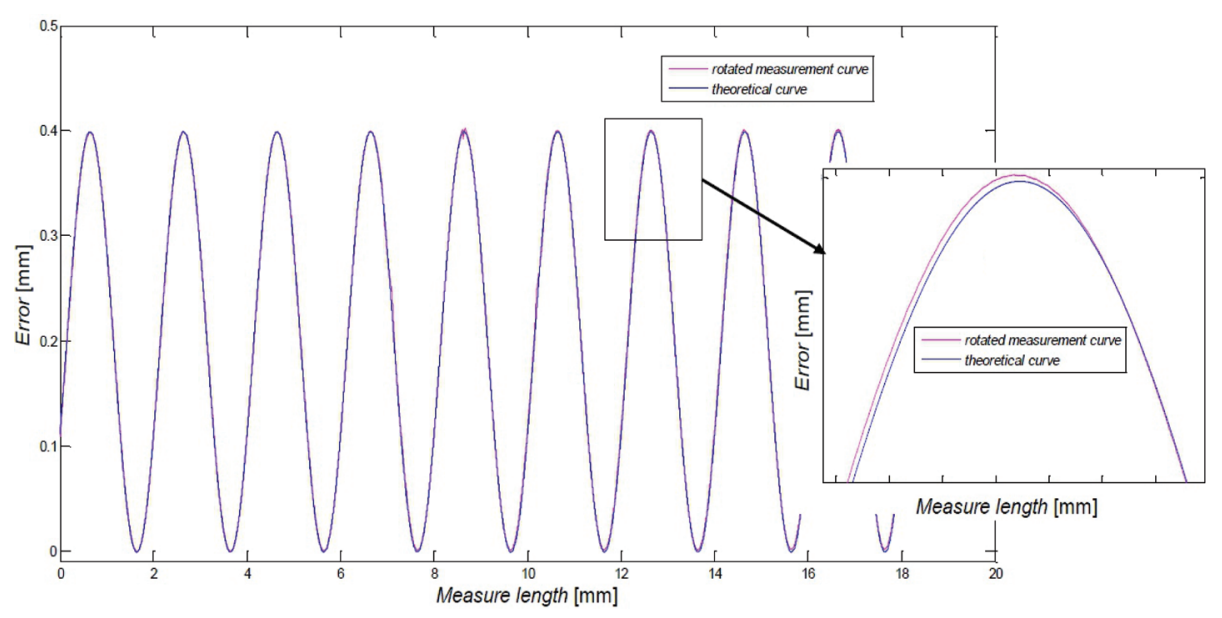

Fig. 10. The rotated measured profile curve and theoretical sinusoidal curve

machined surface was placed under the optical lens of the Contour GT. Then, the adjustment button was rotated forward and backward repeatedly until the interference fringe appeared. After that, the instrument was set to automatic measurement mode. Through automatic measuring the surface of the part and analysis with its corresponding software, the testing result is given in Fig. 9. In Fig. 9a, cutting direction and measuring direction are illustrated. Linear roughness measurement is used to assess the surface quality, the value of surface roughness $(R a)$ along the measuring direction line is about $7.5 \mathrm{~nm}$. In Fig. 9b, areal roughness measurement is used to evaluate the surface quality, the value of surface roughness $(\mathrm{Sa})$ is $4.6 \mathrm{~nm}$. It can be seen that the surface roughness of the machined part meets the requirement of the ultraprecision machining.

The measurement profile of the sinusoidal ring surface was performed by Taylor Hobson. To obtain

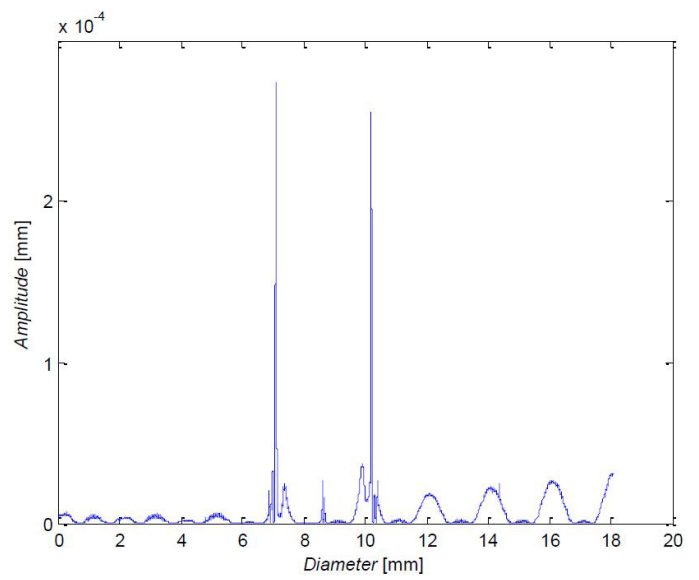

Fig. 11. The result of form accuracy for machined sinusoidal ring surface form accuracy of the machined surface, further analysis for the profile is necessary. Fig. 10 represents the comparison between the measured sinusoidal profile which has been obtained by rotating the original measurement sinusoidal curve and theoretical sinusoidal curve. After post-processing, it can be seen they have a good match. The deviation along the normal direction between the rotated sinusoidal profile and the theoretical one is considered to be the form accuracy, and the result is demonstrated in Fig. 11. It can be observed that the form accuracy PV value $0.274 \mu \mathrm{m}$ is obtained for the machined sinusoidal ring surface with STS.

\section{CONCLUSIONS}

In this paper, the STS assisted diamond turning is applied to machine a sub-millimetre sinusoidal ring surface in ultra-precision. For the purpose of improving the machining precision, several theoretical analyses are implemented before the actual cutting experiment, including tool path generation, tool radius compensation, kinematic analyses of axes, and optimization of the cutting tool parameters. To evaluate the form accuracy of the machined surface, the measured sinusoidal profile is rotated to match the theoretical sinusoidal profile, and the deviation value of two profile curves can be obtained. To verify the validity of theoretical analyses, a sinusoidal ring surface is manufactured with the developed theories. The results of surface roughness and form accuracy of the machined surface are $7.5 \mathrm{~nm}$ in $R a$ and $0.274 \mu \mathrm{m}$ in PV, respectively. The experiment results reveal that the theoretical analyses are effective to improve the machining precision. 


\section{ACKNOWLEDGEMENTS}

This work is supported by the National Key Basic Research and Development Program (973 program) of China (grant no. 2011CB706702), Natural Science Foundation of China (grant nos. 51305161 and 51135006), Jilin Province Science and Technology Development Plan, item (20130101042JC).

\section{REFERENCES}

[1] Zhang, X.D., Fang, F.Z., Wang, H.B., Wei, G.S., Hu, X.T. (2009). Ultra-precision machining of sinusoidal surfaces using the cylindrical coordinate method. Journal of Micromechanics and Micro-engineering, vol. 19, no. 5, p. 054004, DOl:10.1088/0960-1317/19/5/054004.

[2] Wang, Y., Lu, Z., Liu, H., Zhang, H. (2007). Application of freeform surface prism. Infrared and Laser Engineering, vol. 36, no. 3, p. 319-321, D0l:10.3969/j.issn.10072276.2007.03.009. (in Chinese)

[3] Liu Y. (1998). Designing the integrated helmet-mounted display for pilots. Proceedings of SPIE 3560, Display Devices and Systems, p. 175-181, DOI:10.1117/12.319671.

[4] Gao, W., Dejima, S., Yanai, H., Katakura, K., Kiyono, S., Tomita, Y. (2004). A surface motor-driven planar stage integrated with a $\mathrm{XY} \theta \mathrm{Z}$ surface encoder for precision positioning. Precision Engineering, vol. 28, no. 3, p. 329-337, D0l:10.1016/j. precisioneng.2003.12.003.

[5] Jiang, X., Scott, P., Whitehouse, D. (2007). Freeform surface characterization - a fresh strategy. CIRP Annals - Manufacturing Technology, no. 56, no. 1, p. 553-556, DOI:10.1016/j.cirp.2007.05.132.

[6] Gao, W., Araki, T., Kiyono, S., Okazaki, Y., Yamanaka, M. (2003). Precision nano-fabrication and evaluation of a large area sinusoidal grid surface for a surface encoder. Precision Engineering, vol. 27, no. 3, p. 289-298, D0l:10.1016/S01416359(03)00028-X.

[7] Zhang, X.D., Fang, F.Z., Wu, Q.Q., Liu, X.L., Gao, H.M. (2013). Coordinate transformation machining of off-axis aspheric mirrors. The International Journal of Advanced Manufacturing Technology, vol. 67, no. 9-12, p. 2217-2224, Dol:10.1007/ s00170-012-4642-x.

[8] Gong, H., Fang, F.Z., Hu, X.T. (2012). Accurate spiral tool path generation of ultra-precision three-axis turning for non-zero rake angle using symbolic computation. The International Journal of Advanced Manufacturing Technology, vol. 58, no. 9-12, p. 841-847, D0l:10.1007/s00170-011-3433-0.

[9] Lu, H., Lee, D.-W., Lee, S.-M., Park, J.-W. (2012). Diamond machining of sinusoidal grid surface using fast tool servo system for fabrication of hydrophobic surface. Transactions of Nonferrous Metals Society of China, no. 22, suppl. 3, p. 787792, Dol:10.1016/S1003-6326(12)61805-6.

[10] Rasmussen, J.D., Tsao, T.C., Hanson, R.D., Kapoor, S.G. (1994). Dynamic variable depth of cut machining using piezoelectric actuators. Journal of Machine Tool and Manufacture, vol. 34, no. 3, p. 379-392, DOl:10.1016/0890-6955(94)90007-8.
[11] Yin, Z.Q., Dai, Y.F., Li, S.Y., Guan, C.L., Tie, G.P. (2011). Fabrication of off-axis aspheric surfaces using a slow tool servo. International Journal of Machine Tools and Manufacture, vol. 51, no. 5, p. 404-410, Dol:10.1016/j. ijmachtools.2011.01.008.

[12] Yi, A.Y., Li, L. (2005). Design and fabrication of a micro-lens array by use of a slow tool servo. Optics Letters, vol. 30, no. 13, p. 1707-1709, D0I:10.1364/0L.30.001707.

[13] Li, L., Allen, Y.Y., Huang, C., Guan, C.L., Tie, G.P. (2006). Fabrication of diffractive optics by use of slow tool servo diamond turning process. Optical Engineering, vol. 45, no. 11, p. 113401, Dol:10.1117/1.2387142.

[14] Zhu, Z., Zhou, X., Liu, Q., Lin, J.; Zhao, S. (2012). Fabrication of micro-structured surfaces on bulk metallic glasses based on fast tool servo assisted diamond turning. Science of Advanced Materials, vol. 4, no. 9, p. 906-911, D0l:10.1166/ sam.2012.1374.

[15] Wang, Y., Zhao, Q., Shang, Y., Lv, P., Guo, B., Zhao, L. (2011). Ultra-precision machining of Fresnel microstructure on die steel using single crystal diamond tool. Journal of Materials Processing Technology, vol. 211, no. 12, p. 2152-2159, D0I:10.1016/J.jmatprotec.2011.07.018.

[16] Ding, X., Rahman, M. (2012). A study of the performance of cutting polycrystalline Al 6061 T6 with single crystalline diamond micro-tools. Precision Engineering, vol. 36, no. 4, p. 593-603, D0I:10.1016/J.precisioneng.2012.04.009.

[17] Fang, F.Z., Venkatesh, V.C. (1998). Diamond cutting of silicon with nanometric finish. CIRP Annals - Manufacturing Technology, vol. 47, no. 1, p. 45-49, D0l:10.1016/S00078506(07)62782-6.

[18] Rakuff, S., Cuttino, J.F. (2009). Design and testing of a longrange, precision fast tool servo system for diamond turning. Precision Engineering, vol. 33, no. 1, p. 18-25, D0l:10.1016/j. precisioneng.2008.03.001.

[19] Yu, X., Wang, L. (1999). Effect of various parameters on the surface roughness of an aluminum alloy burnished with a spherical surfaced polycrystalline diamond tool. International Journal of Machine Tools and Manufacture, vol. 39, no. 3, p. 459-469, DOI:10.1016/S0890-6955(98)00033-9.

[20] Liu, K., Melkote, S.N. (2007). Finite element analysis of the influence of tool edge radius on size effect in orthogonal micro-cutting process. International Journal of Mechanical Sciences, vol. 49, no. 5, p. 650-660, Dol:10.1016/j. ijmecsci.2006.09.012.

[21] Zong. W.J., Li, D., Cheng, K., Sun, K., Liang, Y.C. (2007). Finite element optimization of diamond tool geometry and cuttingprocess parameters based on surface residual stresses. The International Journal of Advanced Manufacturing Technology, vol. 32, no. 7-8, p. 666-674, D0l:10.1007/s00170-005-0388-z.

[22] Wang, L.,Wang, D., Gao, Y. (2015). Investigations on the effects of different tool edge geometries in the finite element simulation of machining. Strojniški vestnik - Journal of Mechanical Engineering, vol. 61, no. 3, p. 157-166, DOI:10.5545/sv-jme.2014.2051.

[23] Čuš, F., Župerl, U. (2015). Surface roughness control simulation of turning processes. Strojniški vestnik - Journal of Mechanical Engineering, vol. 61, no. 4, p. 245-253, DOI:10.5545/sv-jme.2014.2345. 